\title{
Synthesis and characterization of silver molybdate nanowires, nanorods and multipods
}

\author{
G NAGARAJU, G T CHANDRAPPA* and JACQUES LIVAGE ${ }^{\dagger}$ \\ Department of Chemistry, Central College Campus, Bangalore University, Bangalore 560 001, India \\ ${ }^{\dagger}$ Laboratoire Chimie de la Matière Condensée, Université Pierre et Marie Curie, 4 place Jussieu, \\ 75252 Paris cedex 05, France
}

\begin{abstract}
Silver molybdate nanowires, nanorods and multipods like structures have been prepared by an organic free hydrothermal process using ammonium molybdate and silver nitrate solutions. The powder X-ray diffraction (PXRD) patterns reveal that the silver molybdate belongs to anorthic structure. The thickness, 200-500 $\mathrm{nm}$, for silver molybdate nanorods/wires and 2-5 $\mu \mathrm{m}$ for microrods are identified from SEM images. UV-visible spectrum of silver molybdate nanorods/nanowires shows maximum absorbance at $408 \mathrm{~nm}$. Photoluminescence (PL) spectrum exhibits UV emission at $335 \mathrm{~nm}$, violet emission at $408 \mathrm{~nm}$ and a weak green emission at $540 \mathrm{~nm}$. The influence of hydrothermal synthesis conditions on silver molybdate nanowires, nanorods and multipods compositions were established.
\end{abstract}

Keywords. Silver molybdates; hydrothermal; multipods; nanorods; photoluminescence.

\section{Introduction}

Strategies for obtaining nanosized building blocks such as nanorods, nanowires, nanotubes and nanobelts have received extensive attention because of their distinctive geometries, novel physical and chemical properties and potential applications in nanodevices (Cui et al 2004). Low-dimensional systems represent one of the important frontiers in advanced materials research (Liao et al 2000). Various methods have been developed to synthesize 1-D nanomaterials (Klein et al 1993; Dai et al 1995; Morales and Lieber 1998; Pan et al 2001). In contrast, the solution approaches have been proved to provide an alternative route for the synthesis of 1-D nanomaterials (Buhro et al 1996; Holmes et al 2000; Peng et al 2000; Peng and Peng 2001, 2002). The template method is known to be a very effective method for the fabrication of the desired materials (Torigoe and Esumi 1995; Nagappa et al 2005). However, it is interesting to explore the solution-based synthesis of nanomaterials without the presence of preformed templates (Liao et al 2000; Nagaraju et al 2007). As a simple, cost-effective and efficient synthesis route, the hydrothermal process has been demonstrated as a versatile pathway toward the size and morphology-controllable nanomaterials (Wu et al 2006). Synthesis of 1-D metal molybdates (Guo et al 1995; Liao et al 2000; Mougin et al 2000; Cui et al 2004) have attracted recent interest due to their strong application potential in various fields such

*Author for correspondence (gtchandrappa@yahoo.co.in) as photoluminescence (Kozma et al 2002), microwave applications (Van Uitert and Preziosi 1962), optical fibres (Wang et al 1992), scintillator materials (Wang et al 1994), humidity sensor (Qu et al 2000), magnetic properties (Ehrenberg et al 1997), and catalyst (Driscoll and Ozkan 1994). Lamellar oxide of transition metals, particularly silver molybdate, shows a high temperature friction coefficient without decomposition during melting (Gulbinski et al 2003). These materials have high electrical conductivity and usually have found important applications in conducting glass. For example, silver based superionic glasses and crystals whose conductivity differs by more than 11 orders of magnitude are found to be fast ionic conductors (Adams and Swenson 2000). Most previous approaches for preparation of these families of molybdates need high temperature reaction (Swanson et al 1963). Few reports are related to the synthesis of metal molybdates through solution methods (Sen and Pramanik 2002; Beale and Sankar 2003). Silver molybdates of different phases were traditionally synthesized by the reaction of $\mathrm{MoO}_{3}$ with $\mathrm{Ag}_{2} \mathrm{O}$ (Wenda 1998). Cui et al (2004) prepared silver molybdate nanowires through hydrothermal method using $\mathrm{HCl}$ or $\mathrm{NaOH}$ to control $\mathrm{pH}$ of the solution. Recently, Brechignac et al (2004) prepared silver molybdate clusters by laser annealing method.

In this paper, we report the synthesis of silver molybdate nanorods/nanowires/multipods through a simple hydrothermal approach. In order to understand the habits of one-dimensional silver molybdate nanorods/nanowires/ multipods, the influence of reaction time, temperature and concentration of the precursor on their morphology have been investigated. 


\section{Experimental}

\subsection{Synthesis}

In a typical procedure, $0 \cdot 61 \mathrm{~g}$ ammonium molybdate heptahydrate $\left[\left(\mathrm{NH}_{4}\right)_{6} \mathrm{Mo}_{7} \mathrm{O}_{24} \cdot 4 \mathrm{H}_{2} \mathrm{O}\right]$ was dissolved in $10 \mathrm{~mL}$ distilled water and $0.08 \mathrm{~g}$ silver nitrate was dissolved in $10 \mathrm{~mL}$ distilled water. Then silver nitrate solution was slowly added into ammonium molybdate solution under magnetic stirring to form a homogeneous greenish-yellow precipitate. The resulting precipitate was transferred into a Teflon-lined stainless autoclave. The autoclaves were sealed and maintained at different temperatures (140$200^{\circ} \mathrm{C}$ ) for several hours $(12-72 \mathrm{~h})$. After the hydrothermal process the autoclaves were cooled and the resulting greenish-yellow products were retrieved from the solution by centrifugation, washed with distilled water followed by ethanol to remove the ions possibly remaining in the end product and finally dried in air.

\subsection{Characterization}

Powder X-ray diffraction data were recorded on Philips $X$ 'pert PRO X-ray diffractometer with graphite monochromatized $\mathrm{CuK} \alpha$ radiation $(\lambda=1.541 \AA)$ with Ni filter. The Fourier transform infrared spectrum of the sample was collected using Nicolet FTIR spectrometer. The absorption spectrum of the sample was measured on a UV3101 Shimadzu UV-visible spectrometer. SEM images of

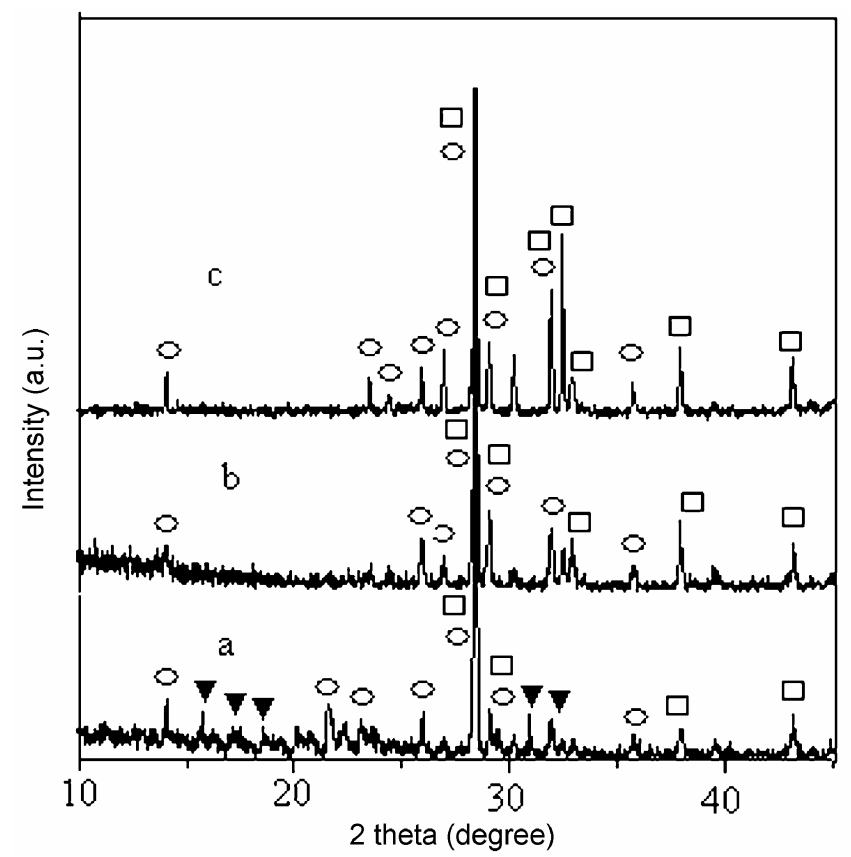

Figure 1. XRD patterns of the products obtained at different conditions: (a) $180^{\circ} \mathrm{C}$ for $24 \mathrm{~h}$, (b) $180^{\circ} \mathrm{C}$ for $72 \mathrm{~h}$ and (c) $200^{\circ} \mathrm{C}$ for $24 \mathrm{~h}$. $\left(\mathrm{O}, \mathrm{Ag}_{2} \mathrm{Mo}_{2} \mathrm{O}_{7} ; \boldsymbol{\nabla}, \mathrm{Mo}_{17} \mathrm{O}_{47} ; \square, \mathrm{Ag}_{6} \mathrm{Mo}_{10} \mathrm{O}_{33}\right)$. the samples were recorded by JEOL JSM-840 A scanning electron microscope. Photoluminescence spectrum was examined at room temperature by Perkin Elmer spectrometer using Xe lamp with an excitation wavelength of $330 \mathrm{~nm}$.

\section{Results and discussion}

Phases and purities of the samples were investigated by PXRD analysis. Figure 1 shows the PXRD patterns of the silver molybdate nanorods/multipods. Hydrothermal treatment of $\left(\mathrm{NH}_{4}\right)_{6} \mathrm{Mo}_{7} \mathrm{O}_{24}$ with $\mathrm{AgNO}_{3}$ led to the formation of mixture of phases including $\mathrm{Ag}_{6} \mathrm{Mo}_{10} \mathrm{O}_{33}$ (JCPDS Card: 72-1689, $a=7 \cdot 59, b=8 \cdot 31, c=11.42 \AA, \alpha=82.6$, $\beta=102 \cdot 9, \quad \gamma=106 \cdot 48), \quad \mathrm{Ag}_{2} \mathrm{Mo}_{2} \mathrm{O}_{7} \quad$ (JCPDS Card: 751505, $a=6.095, b=7.501, c=7.681 \AA, \alpha=110.4, \beta=93.3$, $\gamma=13.58$ ) and $\mathrm{Mo}_{17} \mathrm{O}_{47}$. With increase in temperature, disappearance of $\mathrm{Mo}_{17} \mathrm{O}_{47}$ peaks is observed as shown in figure 1a.

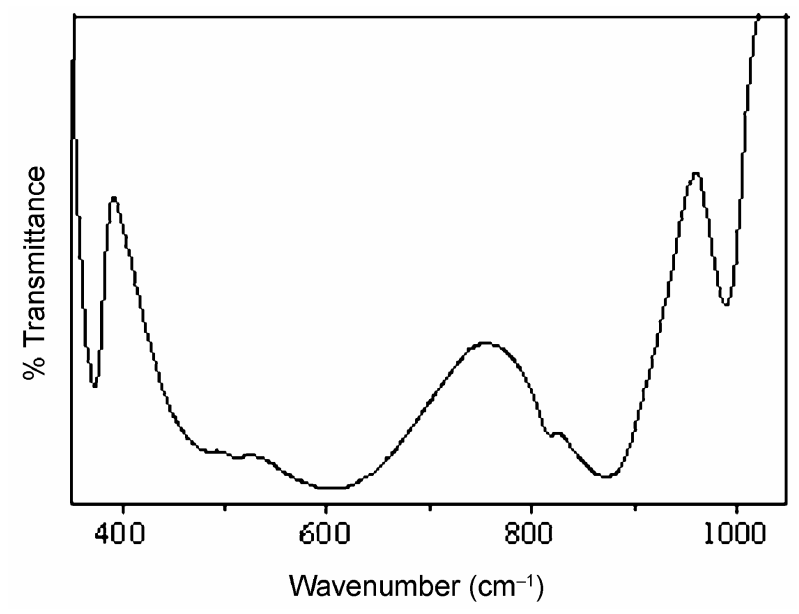

Figure 2. FT-IR spectrum of silver molybdate nanorods prepared at $180^{\circ} \mathrm{C}$ for $48 \mathrm{~h}$.

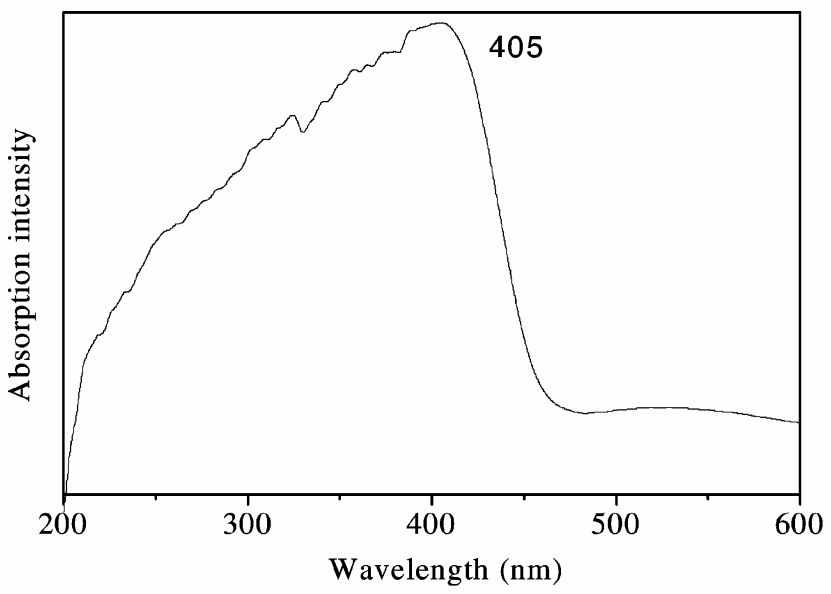

Figure 3. UV-visible spectrum of silver molybdate prepared at $180^{\circ} \mathrm{C}$ for $48 \mathrm{~h}$. 

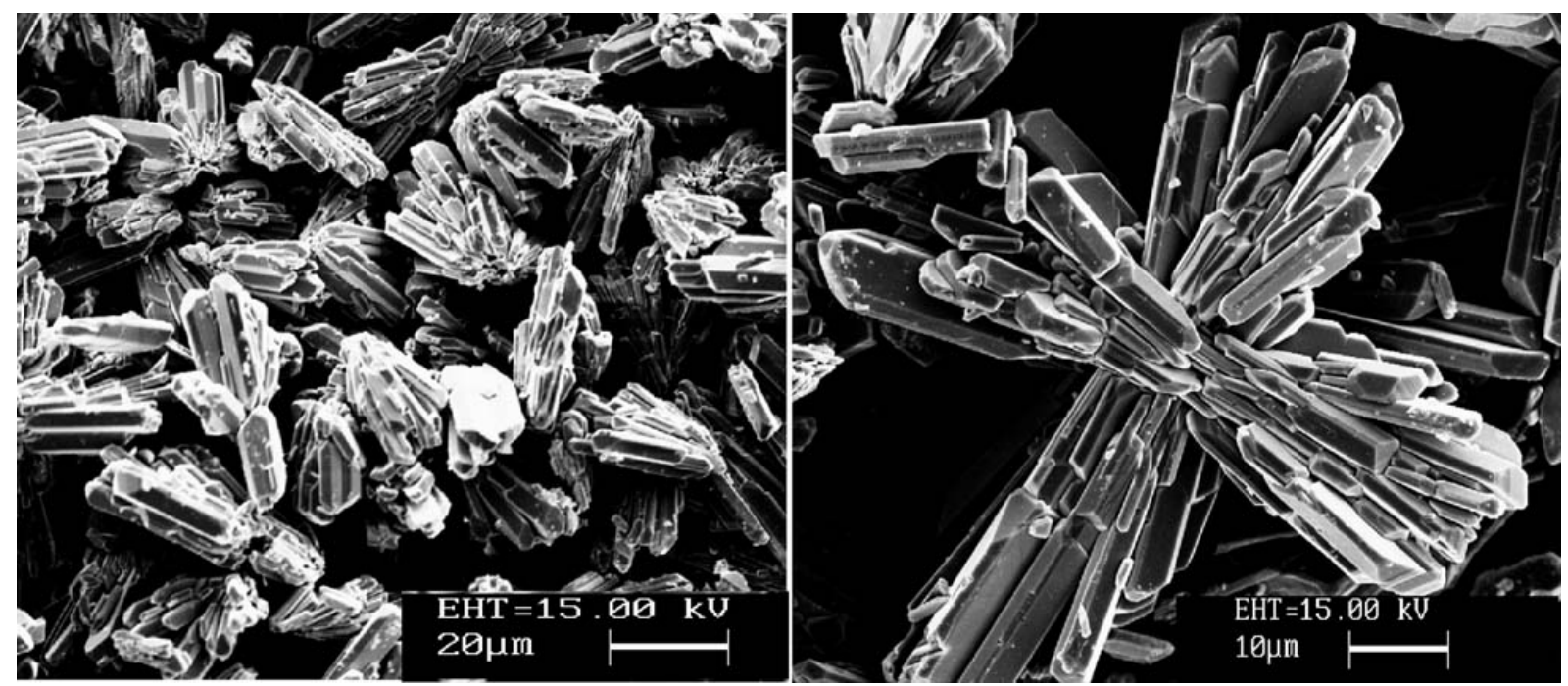

Figure 4. SEM images of the silver molybdate multipods prepared at $180^{\circ} \mathrm{C}$ for $48 \mathrm{~h}$.

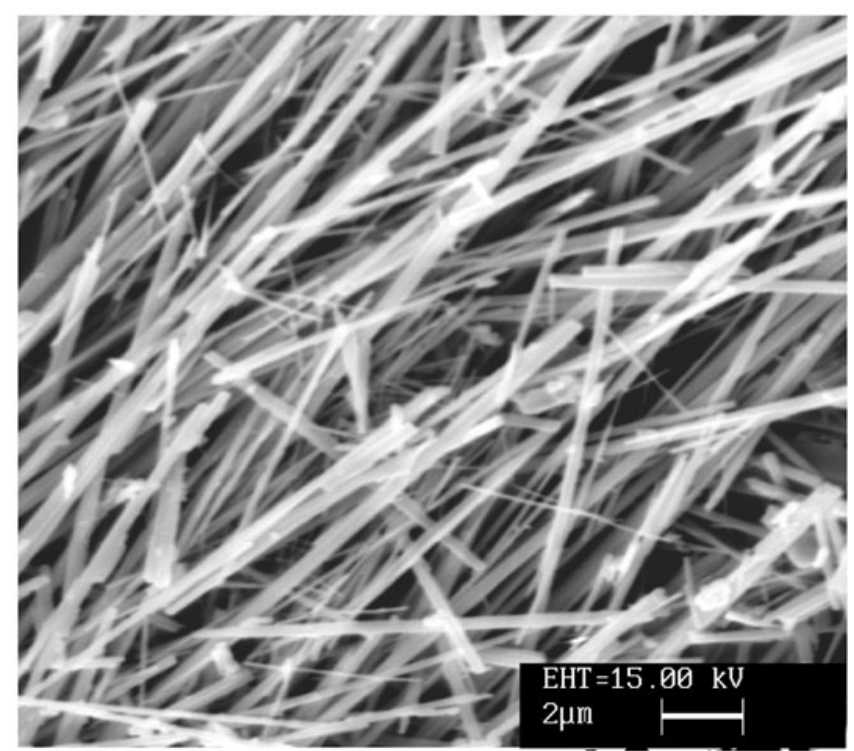

Figure 5. SEM image of silver molybdate nanowires prepared at $200^{\circ} \mathrm{C}$ for $63 \mathrm{~h}$.

Some key structural information was deduced from the FTIR spectrum, which is shown in figure 2. The most important feature is a relatively sharp band at $987 \mathrm{~cm}^{-1}$, characteristic of the stretching of $\mathrm{M}=\mathrm{O}$ double bond which is also found in orthorhombic $\alpha-\mathrm{MoO}_{3}$ indicating a terminal oxygen in the framework. The bands below $1000 \mathrm{~cm}^{-1}$ are assigned as $\mathrm{O}-\mathrm{Mo}-\mathrm{O}$ bond stretching and bending with different Mo-O bond lengths (Guo et al 1995). UV-visible absorption spectroscopy is a useful technique to monitor the size-dependent optical properties of the nanomaterials, due to the quantum confinements of the photo-generated electron-hole carriers in the particles. Nanostructured materials generally exhibit the blue shift behaviour in the absorption measurement due to the size-dependent bandgap structure (Khiew et al 2004). The UV-visible diffuse reflection spectrum of the sample is recorded in the range 200-800 nm on a Shimadzu spectrophotometer, and representative spectrum is shown in figure 3 . It shows that the samples absorb in the spectral region between 250 and $450 \mathrm{~nm}$ and a maximum absorbance around $405 \mathrm{~nm}$ corresponds to a bandgap of $3.05 \mathrm{eV}$.

Figures 4-8 show the SEM morphologies of the sample prepared at $140-200^{\circ} \mathrm{C}$ for $12-72 \mathrm{~h}$. By controlling the experimental conditions (concentration, temperature and duration), we have successfully prepared silver molybdate nanowires, nanorods and multipods of various morphologies through hydrothermal method. SEM images shown in figure 4 shows that the products synthesized at $180^{\circ} \mathrm{C}$ for $48 \mathrm{~h}$ composed of silver molybdate multipods structures, which look like flowers in which each pod radially grows from one centre. A high-magnified SEM image shows that the diameter of each pod of these structures is quite uniform along its length, and the typical diameters of the pods range from 3-6 $\mu \mathrm{m}$. The length of each pod is about 10-30 $\mu \mathrm{m}$. The description of the flower-like silver molybdate structures comes from the geometrical similarity to flowers. Bundles of silver molybdate protrude from the root of the flower-like silver molybdate microrods. In our experiments, it is impossible that the aggregation resulted in the flowerlike structures, because long time ultrasonic treatment could not destroy the structures. Figure 5 shows the SEM morphologies of the sample prepared at $200^{\circ} \mathrm{C}$ for $63 \mathrm{~h}$. The thickness of the nanowires is found to be in the range $200-400 \mathrm{~nm}$ and length is about several micrometers. The thickness of nanowires is found to be almost uniform along its entire length. By doubling the concentration of any one of the reactants (ammonium molybdate and silver nitrate), silver molybdate nanorods 


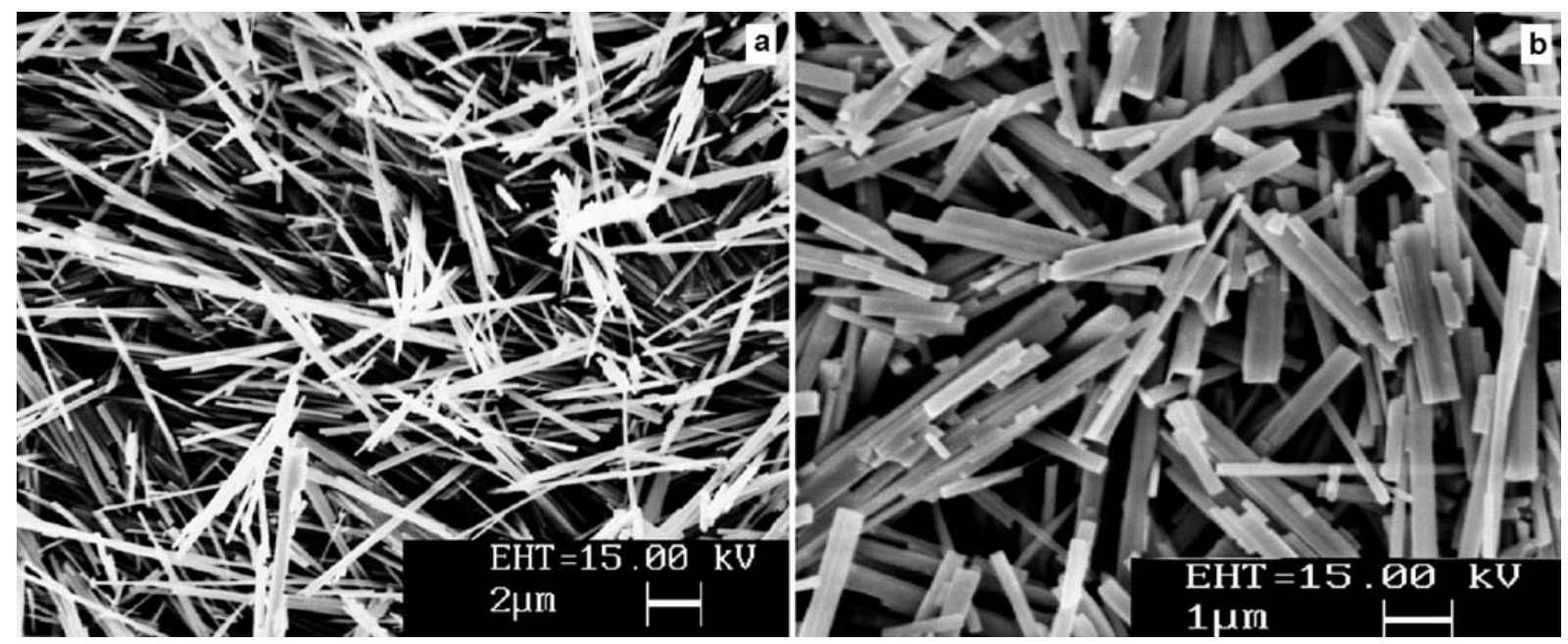

Figure 6. SEM images of silver molybdate nanorods prepared at $180^{\circ} \mathrm{C}$ for $48 \mathrm{~h}$ by doubling the concentration of both (a) ammonium molybdate and (b) silver nitrate.
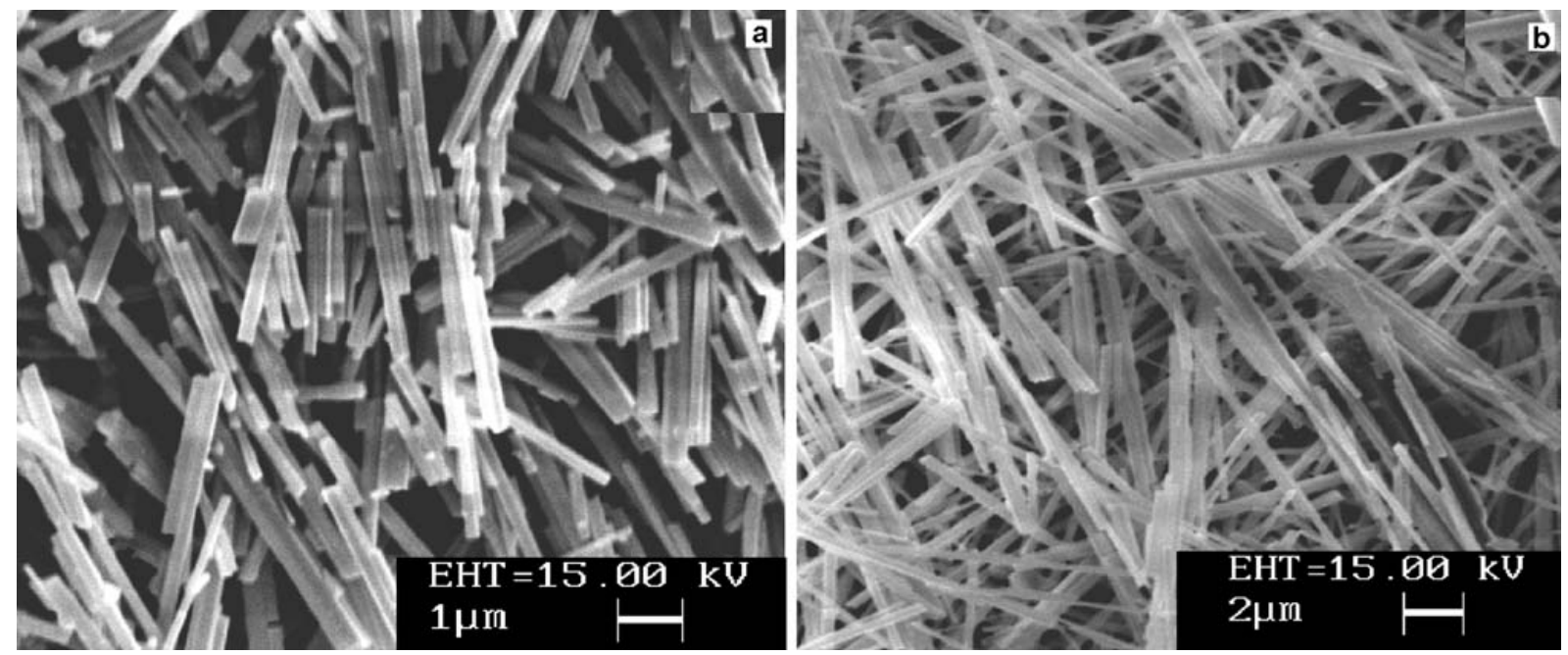

Figure 7. SEM images of the silver molybdate nanorods and nanowires prepared at (a) $140^{\circ} \mathrm{C}$ for $24 \mathrm{~h}$ and (b) $140^{\circ} \mathrm{C}$ for $12 \mathrm{~h}$.

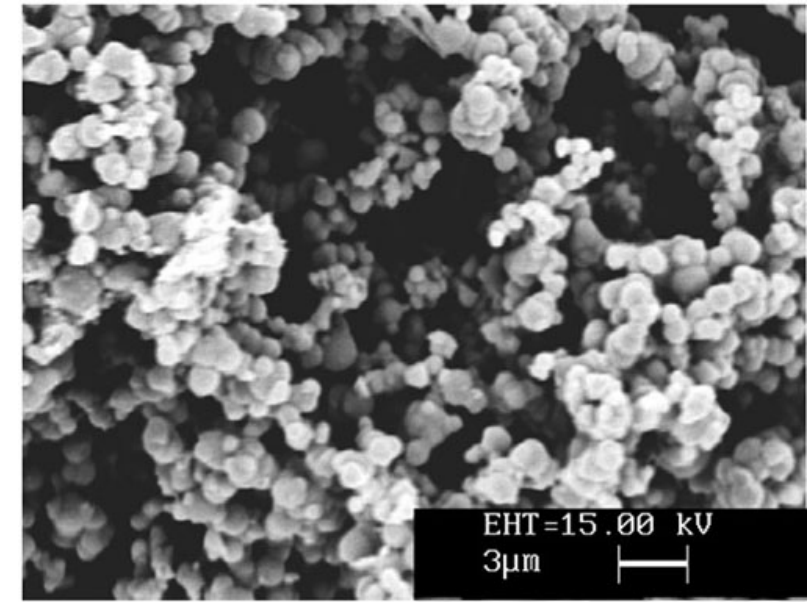

Figure 8. SEM image of silver molybdate nano/micro spheres obtained at $180^{\circ} \mathrm{C}$ for $48 \mathrm{~h}$ by dissolving silver nitrate in $10 \mathrm{~mL}$ ethyl alcohol. (figures 6a and b) of uniform diameter of about 200$500 \mathrm{~nm}$ are obtained at $180^{\circ} \mathrm{C}$ for $48 \mathrm{~h}$. When both temperature and treatment time are reduced to $140^{\circ} \mathrm{C}$ for 24 and $12 \mathrm{~h}$, by keeping concentration of the precursor constant, nanorods and nanowires of about 300-600 nm diameter are obtained as shown in figures $7 \mathrm{a}$ and $\mathrm{b}$. Silver nitrate crystals are dissolved in $10 \mathrm{ml}$ ethyl alcohol instead of water by keeping all the above parameters the same and nano/micro spheres are obtained as shown in figure 8 .

In order to assess the crystalline quality and investigate the optical properties of the silver molybdate micro/ nanorods, PL spectrum of $320 \mathrm{~nm}$ is taken using Xe lamp as the excitation source. Figure 9 shows photoluminescence spectrum of the sample prepared at $180{ }^{\circ} \mathrm{C}$ for $48 \mathrm{~h}$. It is generally assumed that the measured emission spectrum of silver molybdate complex is mainly attributed to the charge-transfer transitions within the molybdate complex. It shows UV emission at $335 \mathrm{~nm}$, violet 


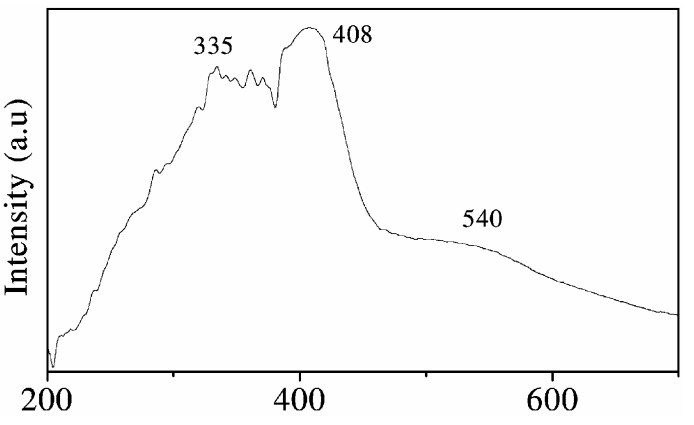

Figure 9. Photoluminescence spectrum of silver molybdate multipods.

emission at $408 \mathrm{~nm}$ and a weak green emission at $540 \mathrm{~nm}$. The emission in the UV region is attributed to the recombination between electrons in the conduction band and holes in the valence band (Gao et al 2006). Violet emission at $408 \mathrm{~nm}$ is attributed to the exciton transition (Wang and Gao 2003). Weak and broad green emission at $540 \mathrm{~nm}$ can be ascribed to a defect structure. The strong UV emission and weak green emission in PL spectrum indicate that the silver molybdate nano/microrods have a good optical quality with few oxygen vacancies (Monticone et al 1998). Oxygen vacancies have been considered as the most common defects and usually act as radiative centres in luminescence process. In addition, silver molybdate with high aspect ratio should also favour the existence of large quantities of oxygen vacancies.

\section{Conclusions}

In summary, by means of an organic-free hydrothermal process, silver molybdate nanowires, nanorods and multipods like structures have been successfully prepared. It is found that concentration of the precursor, reaction temperature and treatment time have significant influence on the morphology of silver molybdate nanowires, nanorods and microrods. PXRD results show the formation of mixed phases of silver molybdates. SEM images reveal that the thickness of the nanowires/rods is in the range of $200-500 \mathrm{~nm}$ and microrods are in the range of 3-6 $\mu \mathrm{m}$. UV-vis spectrum of silver molybdate nanorods/nanowires shows maximum absorbance at $405 \mathrm{~nm}$ corresponding to a bandgap of $3.05 \mathrm{eV}$. The PL spectra of the silver molybdate microrods consist of UV emission at $335 \mathrm{~nm}$ and violet emission at $408 \mathrm{~nm}$.

\section{Acknowledgements}

One of the authors (GTC) is thankful to the Department of Science and Technology, NSTI Phase-II, New Delhi, Government of India, for financial support to carry out the research work. We also thank the Central Facility, Department of Physics, and Metallurgy, Indian Institute of Science, Bangalore, for their help with XRD studies and SEM images.

\section{References}

Adams S and Swenson J 2000 Phys. Rev. Lett. 844144

Beale A M and Sankar G 2003 Chem. Mater. 15146

Brechignac C, Ph. Cahuzhac, Kebaili N, Lando A, Masson A and Schmidt M 2004 J. Chem. Phys. 1219617

Buhro W E, Hickman K M and Trentler T J 1996 Adv. Mater. 8685

Cui Xianjin, Yu Shu-Hong, Li Lingling, Biao Liu, Li Huabin, Mo Maosong and Liu Xian-Ming 2004 Chem. Eur. J. 10 218

Dai H, Wong E W, Lu Y Z, Fan S and Lieber C 1995 Nature 375769

Driscoll S and Ozkan U S 1994 Stud. Surf. Sci. Catal. 82 367

Ehrenberg E, Weitzely H, Heidy C, Fuessy H, Wltschekz G, Kroenerx T, van Tol J and Bonnet M 1997 J. Phys. Condens. Matter 93189

Gao Peng, Ying Chen, Wang Shuqing, Ye Lina, Guo Qixun and Xie Yi 2006 J. Nanopart. Res. 8131

Gulbinski W, Suszko T, Sienicki W and Warcholinski B 2003 Wear 254129

Guo Jingdong, Zavalij Peter and Stanley Whittingham M 1995 J. Solid State Chem. 117323

Holmes J D, Johnston K P, Doty R C and Korgel B A 2000 Science 2871471

Khiew P S, Radiman S, Huang N M and Md. Soot Ahmad 2004 J. Cryst. Growth 268227

Klein J D, Herrick R D, Palmer D, Sailor M J, Brumlik C J and Martin C R 1993 Chem. Mater. 5902

Kozma P, Bajgar R and Kozma J P 2002 Radiat. Phys. Chem. 34127

Liao Hong-Wei, Wang Yan-Fei, Liu Xian-Min, Li Ya-Dong and Qian Yi-Tai 2000 Chem. Mater. 122819

Morales A M and Lieber C M 1998 Science 279208

Mougin Oliver, Dubois Jean-Luc, Mathieu Francois and Rousset Abel 2000 J. Solid State Chem. 152353

Monticone S, Tufeu R and Kanaev A V 1998 J. Phys. Chem. B102 2854

Nagappa B, Chandrappa G T and Livage J 2005 Pramana-J. Phys. 65917

Nagaraju G, Tharamani C N, Chandrappa G T and Livage J 2007 Nanoscale Res. Lett. 2461

Pan Z W, Dai Z R and Wang Z L 2001 Science 2911947

Peng X G, Manna L, Yang W D, Wickham J, Scher E, Kadavanich A and Alivisatos A P 2000 Nature 40459

Peng Z A and Peng X G 2001 J. Am. Chem. Soc. 1231389

Peng Z A and Peng X G 2002 J. Am. Chem. Soc. 1243343

Qu W, Wlodarski W and Meyer J -U 2000 Sens. Actuators B64 76

Sen A and Pramanik P 2002 Mater. Lett. 52140

Swanson H E, Morris M C, Stinchfield R P and Evans E H 1963 NBS Monogr. 2524

Torigoe K and Esumi K 1995 Langmuir 114199

Van Uitert L G and Preziosi S 1962 J. Appl. Phys. 332908

Wang H, Medina F D, Zhou Y D and Zhang Q N 1992 Phys. Rev. B45 10356

Wang H, Medina F D, Liu D and Zhou Y D 1994 J. Phys. Condens. Matter 65373

Wang Jinmin and Gao Lian 2003 J. Mater. Chem. 132551

Wenda E J 1998 J. Therm. Anal. Calorim. 53861

Wu Jianbo, Zhang Hui, Du Ning, Ma Xiangyang and Yang Deren 2006 J. Phys. Chem. B110 11196 